\title{
Capability of THz for Thermo-transmittance/water content measurements of insulating materials: heat and mass transfer.
}

\author{
M. BENSALEM ${ }^{*}$, A. SOMMIER* ${ }^{*}$ J. C. Mindeguia**, J. C. BATSALE ${ }^{*}$ and C. PRADERE ${ }^{*, * * *}$ \\ * I2M, UMR CNRS 5295, Esplanade des Arts \& Métiers, 33405 Talence, France. \\ ** I2M, UMR CNRS 5295, Université de Bordeaux, 351 cours de la Libération, 33405 Talence, France. \\ ${ }^{* * *}$ Tomsk polytechnic university, Lenin Av 30, Tomsk 634050, Russia.
}

\begin{abstract}
$\mathrm{THz}$ waves show to be an effective mean for several applications such as security, non-destructive, water content monitoring for wood and food products. The increasing use of $\mathrm{THz}$ is due to the sufficient resolution for such applications, its low cost and the safe levels of radiation. This study consists of two parts. The first one represents the use of $\mathrm{THz}$ imaging technique for studying the transient thermal transfer within semi-transparent insulating materials. The second part will be dedicated to transient hydric transfer. detailed.

The developed setup with appropriate image processing methods linked to the lock-in amplification mode will be
\end{abstract}

\section{Introduction}

Recently, the use of non-contact methods for measuring temperature and water content in several applications has been extensively developed. As the characteristic time of thermal transfer is shorter than the hydric one (few minutes), a high acquisition imaging $\mathrm{THz}$ system allowing the measurements at thermal transient case has to be developed.

For thermal applications, Non-contact methods for temperature measurement have been recently developed [1]. Infrared thermography showed a capability to determine the thermal transmittance of certain solids which allows measuring full-field temperature at steady-state (at given temperature) [1]. The infrared imaging technique showed to be efficient to evaluate the heat transport properties in chemical reaction at micro scale [2]. When the sample is opaque in visible or infrared range, $\mathrm{THz}$ could be an efficient alternative tool for non-contact temperature imaging [3]. Therefore the 2D and 3D temperature distribution could be reconstructed by tomography [4].

$\mathrm{THz}$ waves have been rarely applied as a tool for temperature measurement [3]. C. Pradere et al used $\mathrm{THz}$ source with infrared mono-detector to estimate the thermal reflectance coefficient for metal and semiconductor materials. The technique allowed the estimation of a punctual thermo reflectance at transient temperature through recording the reflectance during heating. It has been shown that at millimeter wavelength range, the coefficients are 100 times higher than their values in visible range (sensitivity). In [5], C. Poulin et al investigated the capability of THz to measure the thermo-transmittance of polymer material subjected to a thermal gradient. The 2D transmitted signal coupled with a simulated 3D temperature distribution allowed the estimation of the thermo-transmittance coefficient of POM.

On the hand, THz technique is highly involved in water content measurement in porous biomaterials. It has been shown that $\mathrm{THz}$ represents an effective tool for such measurements due to the high absorptivity of water at millimeter wavelength range. Contrary to harmful radiations (X-Ray or gamma-Ray [6]), THz represents a safe technique due to its non-ionizing radiations. Moreover, $\mathrm{THZ}$ is cheap comparatively to other technique of water content measurements such as Neutron Imaging Radiography [7]. THz is also a technique that can be implemented in in-line industry process and the capability to scan big surface contrary to Magnetic Resonance Imaging technique [8].

It is shown in [9] that the water transport during drying processes represents a key stage in wood-based material industry, the not homogeneous drying can lead to the spread of cracks within the material and therefore providing low quality final product. Numerical simulations of water movement based on hygro-mechanical behavior of wood highlight the anisotropic shrinkage coefficients needs the spatial distribution of water content within wood. In previous study [10], the author investigated the capability of $\mathrm{THz}$ imaging technique to map the water content within wood as steady-state case (Wood contains known water content that corresponds the water content of equilibrium after $b$ conditioning at given relative humidity)

$\mathrm{THz}$ radiations are electromagnetic waves located between infrared and microwaves (30-3000 $\mu \mathrm{m})$. At $\mathrm{THz}$ frequencies, the waves can penetrate through insulation materials but are reflected by metals and absorbed by water. These limitations make $\mathrm{THz}$ technology an effective tool for security applications such as the detection of chemical weapons, metallic and non-metallic weapons, flammables and Non Destructive Testing [11]. THz radiation has been also used for quality inspection of plastic and polymer-wood composite, food (i.e., dried food, wheat grain, coffee leaves, dry fruit, metallic contamination in chocolate products, etc.) $[12,13]$, plant breeding [14], paper quality [15] and in paint and art preservation applications [16].

In this paper, the first part will be dedicated to study the efficiency of $\mathrm{THz}$ imaging technique for contactless temperature measurement at transient case for homogeneous and heterogeneous semi-transparent materials. First, 
measurement system settings allowing fast imaging of transmittance based on infrared camera coupled with THz-tothermal conversion (TTC) in lock-in amplification mode is detailed. Then, a Beer-Lambert-based calibration between THz transmittance and transient temperature measurement taken by thermocouple on material surface (PVC, PTFE and wood will be carried out. The use of temperature of surface in calibration will be justified. Then, the thermo-transmittance coefficient of a given pixel will be estimated using an inverse method. Finally, using the identified parameter with an appropriate image processing, the temperature image will be reconstructed which allows validating $\mathrm{THz}$ technique as contactless method for temperature measurement in transient case.

However, the second part will be devoted to show the efficiency of the THz technique in monitoring of mass diffusion within a homogeneous sample (blotting paper). First, Beer-Lambert law-based model linking the absorption coefficient of blotting paper and its water content is used. Finally, minimization based on Levenberg-Marquardt algorithm between an obtained experimental image and numerical simulation (central differencing scheme) allows the estimation of diffusion coefficient of blotting paper. In fact, the model representing the water content as function of transmittance is used in [10] within a wood sample using infrared mono-detector.

In this study, a new contactless technique for temperature measurements of $\mathrm{THz}$ semi-transparent materials during transient thermal transfer is developed. The results show that $\mathrm{THz}$ technique could be suitable for applications aiming to 3D contactless temperature reconstruction (transient thermal tomography) that will be the aim of future study. On the other hand, the $\mathrm{THz}$ shows efficiency for monitoring the water vapor diffusion and estimating of diffusion coefficient of blotting paper.

\section{Analytical models}

For thermal transfer, the used Beer-Lambert based model is given as follows:

$$
\Gamma(x, y, \lambda, T)=-\log \left(\frac{I(x, y, \lambda, T)}{I_{0}\left(x, y, \lambda, T_{0}\right)}\right)=\mu(\lambda, T) \cdot e
$$

Where: $\Gamma$ is the $\mathrm{THz}$ thermo-transmittance of a pixel at a given temperature $T$ in $(K), I$ is the transmitted signal at temperature $T$ in $(D L), I_{0}$ is the signal in $(D L)$ at reference temperature $T_{0}$ in $(K), \mu(\lambda, T)$ is absorption coefficient in $\left(m^{-1}\right), e$ is thickness $\left(\mathrm{m}^{-1}\right)$ and $\lambda$ is wavelength in $(m)$. Thus, for each pixel and for given wavelength, the Eq. (1) can be written as follows:

$$
-\log \left(\frac{I(T)}{I\left(T_{0}\right)}\right)=e \frac{\partial \mu(T)}{\partial T} d T=\kappa \cdot e\left(T(z)-T_{0}\right)
$$

Where: $k$ is the thermo-transmittance coefficient $\left(\mathrm{m}^{-1} K^{1}\right)$. On the other hand, the hypothesis of lumped body allows taking the temperature in Eq. (2) as independent of $z$. We can assume that the thermal transfer in the thickness is negligible and the sample is releasing temperature in equal manner overall. This behavior has been checked by calculating the Biot number of studied samples that found to be less than 1. Moreover, the temperature measured by thermocouple at one pixel in Region Of Interest is supposed equal all over the sample. Therefore, in transient case, the transmittance temperature-dependency is given by:

$$
\Gamma(T)=\kappa e\left(T-T_{0}\right)
$$

As the thermo-transmittance is linear with temperature gradient, the estimation of the coefficient of thermotransmittance $\kappa$ is proportional to the slope.

For the hydric diffusion, the used model given in [10] considering water content as fictitious layer $\left(e_{w}\right)$ allows writing at ambient temperature:

$$
\frac{-\log \left(\frac{I(x, y, \lambda)}{I_{0}(\lambda)}\right)}{e_{s}}=\mu_{s}+\mu_{w} \frac{e_{w}}{e_{s}} W
$$


Where: I is the transmitted signal in $(D L)$ at water content $W$ in ( $\mathrm{kg}$ of water/.kg of dry material), $I_{0}$ is the signal in $(D L)$ at reference hydric state $\left(W_{0}=0\right), \mu_{s}$ and $\mu_{w}$ are the absorption coefficients of solid and water in $\left(m^{-1}\right), e_{s}$ and $e_{w}$ are the thicknesses of solid and water in $(m)$.

The Eq. (4) representing a linear relationship between the global water content and the absorbance of wet sample allowed estimating the absorption coefficient and density of dry sample knowing the water content. In this work, water content measurement allowing the estimation of diffusion coefficient of blotting paper by knowing the absorption coefficient and density of dry sample.

\section{Experimental setup}

The experimental setup consists of (1) a THz source (2) an infrared camera with TTC and lenses. The THz wave source is a Gunn diode (Virginia diodes, Inc) with a power of $200 \mathrm{~mW}$ wrking at frequency of $110 \mathrm{GHz}$ (equivalent of wavelength $2.7 \mathrm{~mm}$ ). The detector consists of InSb quantum detector-based infrared camera (FLIR SC7000) with a spectral band of $3.5 \mu \mathrm{m}-5.15 \mu \mathrm{m}$ and acquisition frequencies up to $1 \mathrm{kHz}$ and $\mathrm{THz}$-to-thermal converter that is placed at the camera focal plane. The TTC consists of a carbon film absorbs THz radiation which leads to a thermal diffusion detected by infrared camera [17].

TA beam expander composed of two plano-convex lenses made of PTFE (THORLABS) with diameter of 110 $\mathrm{mm}$ and focal distance of $100 \mathrm{~mm}$. The TA expander situated between the source and the sample allows expanding the $\mathrm{THz}$ beam and minimizing the radial intensity variation on the sample plane. The transmitted beam is imaged on the TTC through a THz objective consists of three PTFE lenses (Focus).

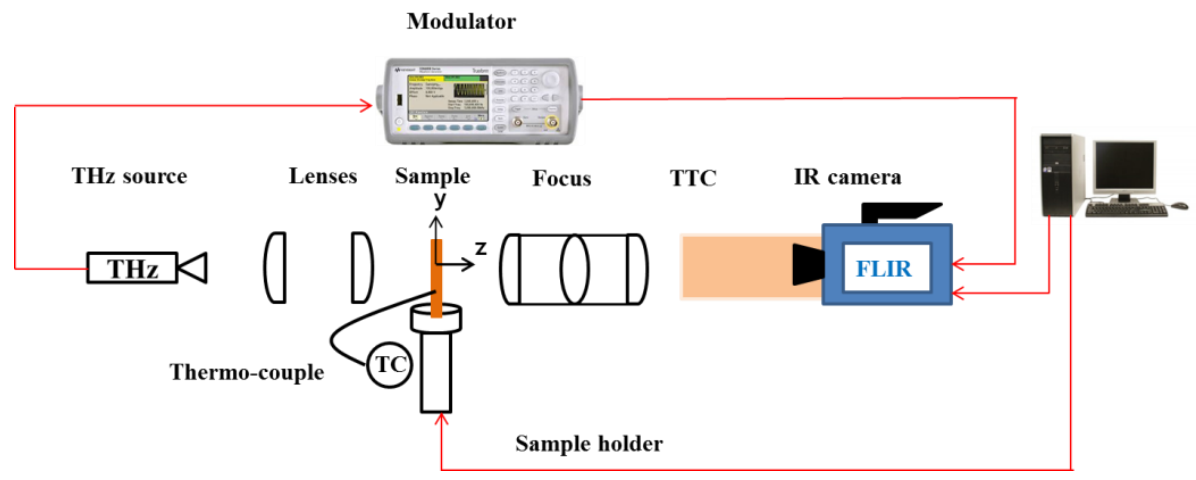

Fig. 1: Experimental setup for transient thermal and hydric diffusion.

The sizes of the samples were as follows: PVC $(100 \mathrm{~mm} \times 40 \mathrm{~mm} \times 4 \mathrm{~mm})$, PTFE $(100 \mathrm{~mm} \times 40 \mathrm{~mm} \times 6 \mathrm{~mm})$ and wood $(100 \mathrm{~mm} \times 40 \mathrm{~mm} \times 3 \mathrm{~mm})$. The samples were pre-heated in an oven at $100^{\circ} \mathrm{C}$. Once the temperature of sample is homogenized (checked by thermocouple at different positions), it was then placed on the focal plane as shown in the (figure 1). A thermocouple (type K) was fixed in the center of sample on the side in front of the $\mathrm{THz}$ source to record its temperature release by natural convection during experiment until it reached the ambient temperature (approximately $20^{\circ} \mathrm{C}$ ). It is shown the transient evolution of temperature of each sample affected the capacity of the material to transmit $\mathrm{THz}$ waves.

A wave-form generator (Agilent 33500B Series) was used to synchronize the THz source ignition and the CCD acquisition [17]. The synchronized signals represent the square signal ON/OFF phase of the $\mathrm{THz}$ source and square signal triggering the camera (figure 2). The generator also allowed for the modulation of the excitation of the $\mathrm{THz}$ beam with the onset of filming to limit diffusion on the TTC $x-y$ plane and obtain sufficient signal. Thus, during filming, the duty cycle was $50 \%$ which (source is turned on only for a half filming duration). In fact, when the duty cycle is greater than $50 \%$, the relaxation of material will not be sufficient (the response of the material to the excitation will be chopped off). An integration time (IT) of $1400 \mu \mathrm{s}$, frequency acquisition of $200 \mathrm{~Hz}$, filming duration of $1 \mathrm{~s}$, inter-filming duration of $2 \mathrm{~s}$, pixel size of $250 \mu \mathrm{m}$ and film number of 300 films were chosen for this study. The synchronization process is sketched in figure 2 . 


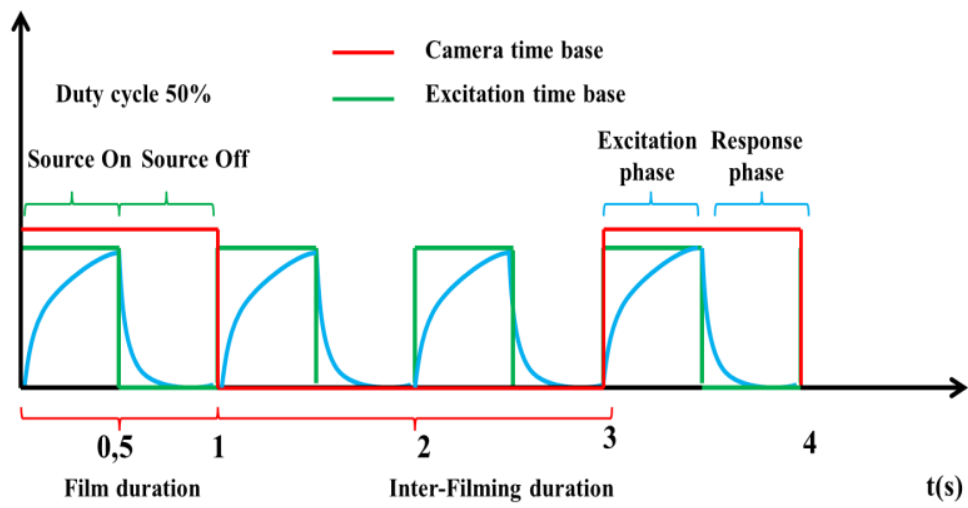

Fig. 2: Synchronization of source excitation frequency with acquisition frequency.

For the transient hydric transfer (diffusion), in addition of the set-up used for measurement of temperature (figure 1), a fluidic exciter has been used. The fluidic exciter consists of: (1) enclosure, (2) aquarium pump and (3) saline solution of $\mathrm{RH}$ of $97 \%$. The enclosure is $\mathrm{THz}$ semi-transparent plastic material allows the insulation of the two lateral faces of sample. The sample is insulated from all sides by rubber material except the excitation face. The aquarium pump ensures the circulation of vapor next to the non-insulated side of sample. The humidity is checked by $\mathrm{RH}$ sensor placed on the output of steam tube. The exciter is shown in the figure 3.

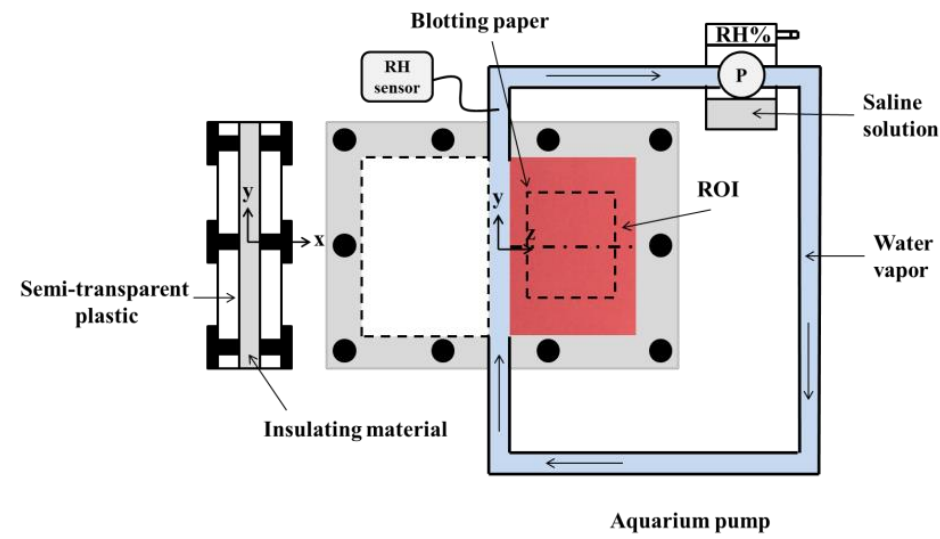

Fig. 3: Excitation of homogeneous sample (blotting paper).

For the diffusion experiment, the fixed parameters are the same parameters mentioned above except the interfilming duration. As the hydric diffusion is long, the inter-filming duration is fixed at 5 min, film number that is 200 films. Finally, the obtained results represent a series of films of duration of 1 second of 200 images for each film. Therefore, the extracting of information needs an appropriate image processing. The next paragraph will be dedicated to the used processing methods of obtained data.

\section{Post-processing}

The obtained films of transmitted $\mathrm{THz}$ signal during a decrease of temperature in each sample represent raw data that have to be processed. As the experiment is carried out in lock-in mode with synchronization of filming and excitation, the films represent a periodic response of material (bleu curve in figure 2). Therefore, to extract the amplitude of transmitted signal image from each film (period), four-image algorithm processing method [18] was applied. The four image algorithm showed better image quality after comparison with other methods of amplitude extracting such as Fourier spectral analyze, least-squares minimization and synchronous detection.

As shown in figure 3, the source was modulated to $1 \mathrm{~Hz}$ with an acquisition frequency of $200 \mathrm{~Hz}$ (image/film), and duration of $1 \mathrm{~s}$. The image size is $128 \times 160$ pixels resulting in enormous amount of data $(128 \times 160 \times 200 \times 300)$. First, an ROI was chosen (approximately $30 \times 80$ pixels). The four-image algorithm is a method based on the integration of signal along each quarter of period as shown in Eq. (7). 


$$
\left.S_{i}\right|_{i=1,2,3,4}=\int_{\frac{(i-1) T}{4}}^{\frac{i T}{4}} A(x, y, t) d t
$$

$S_{i}$ is the integrated signals at each quarter of the period in $(D L)$, and $A$ is the signal of each pixel at instant $t$. Thus, the amplitude for each period can be given as follows:

$$
I(x, y)=\frac{1}{N p / 4} \sqrt{\left(S_{1}-S_{3}\right)^{2}+\left(S_{2}-S_{4}\right)^{2}}
$$

$\mathrm{Np}$ is the number of points per period (in this study corresponds to acquisition frequency of $200 \mathrm{~Hz}$ ). The obtained signal $I$, represents the image of the amplitude per film. The obtained images represent the image of signal amplitude that showed to be higher quality compared to raw data. However, the images of amplitude need further processing to decrease the present noise. Hence, in order to diminish the noise that could be incoming from the infrared camera and temperature fluctuations, Singular Value Decomposition (SVD) was applied [17].

The SVD method is a processing method consisting in the decomposition of obtained amplitude images. First, amplitude cubes $(30 \times 80 \times 300)$ were reshaped to an image size $(30 \times 80) \times 300$ to obtain matrix $M$ containing spacetime information. Second, projection on separated bases of space and time was carried out as follows:

$$
M(s, t)=U(s) \cdot S(r) \cdot V^{\prime}(t)
$$

The matrix $M(s, t)$ is the Matrix space-time of amplitude images, $U(s)$ is the base containing information on space $s, V(t)$ is the transposed base containing information on time $t$ and $S(r)$ is a diagonal matrix containing the non-null decreasing modes values $r$ (singular values). The first values of matrix $S$ contain the signal information, where the remaining values represent the noise in the images. Therefore, the matrix $M(s, t)$ can be reconstructed according to Eq. (7) by keeping only the truncated $r^{*}$ modes of $S$ non-null.

Moreover, in the SVD processing method, deleting most modes can clearly reduce the noise but changes also the shape of the signal, while deleting only few modes would not sufficiently reduce the noise signal. Thus, a compromise should be made between maintaining the shape of the signal and reducing noise. Therefore, to determine the necessary number of modes to keep non-null, plotting the modes on a logarithmic scale is suggested. Thus, the number of modes corresponding to the breakpoint represents the number of modes used to reconstruct cleaned-up images without losing information of signal at each pixel.

\section{Results}

\subsection{Thermo-transmittance}

The results showed that for a given pixel the transmitted signal increases with decrease of temperature and tends to stabilize when the temperature sample attains the ambient temperature. The figures $(4 \mathrm{a}, 4 \mathrm{c}$ and $4 \mathrm{e})$ show the transmittance of a pixel and temperature measurements taken by thermocouple function of time. Two homogeneous semi-transparent materials (PVC and PTFE) and heterogeneous material (maritime pine woos) are represented. The figures show that the transmittance variation is low and around 0.13 for $40^{\circ} \mathrm{C}$ for PCV, 0.12 for $50^{\circ} \mathrm{C}$ for PTFE and 0.07 for $20^{\circ} \mathrm{C}$ for wood. Contrary to the high sensitivity of THz waves to hydric state of sample (from $100 \%$ of transmitted signal through a dry sample to $0 \%$ of transmitted signal through completely wet sample), the sensitivity of $\mathrm{THz}$ to temperature is low (from $12 \%$ of transmittance through hot sample to $0 \%$ through sample at relative temperature $0{ }^{\circ} \mathrm{C}$ ). Therefore, this shows the capacity of setup to detect such small sensitivity. Moreover, the transient case duration is different from sample to other (shorter for wood) due the constant of characteristic time of lumped body equation solution. Therefore, the steady state is attained quickly in wood. The lumped body hypothesis supposing that the punctual temperature measurement taken by thermocouple is the same over the ROI is proven by calculating Biot number and by 3D Transient Finite Volume numerical simulations [19]. This hypothesis justifies the calibration of thermotransmittance of a given pixel and temperature measured by thermocouple in ROI as shown in figures (4b, 4d and $4 \mathrm{f})$. The chosen pixel is situated in the ROI where the signal is sufficiently high but not saturated. 


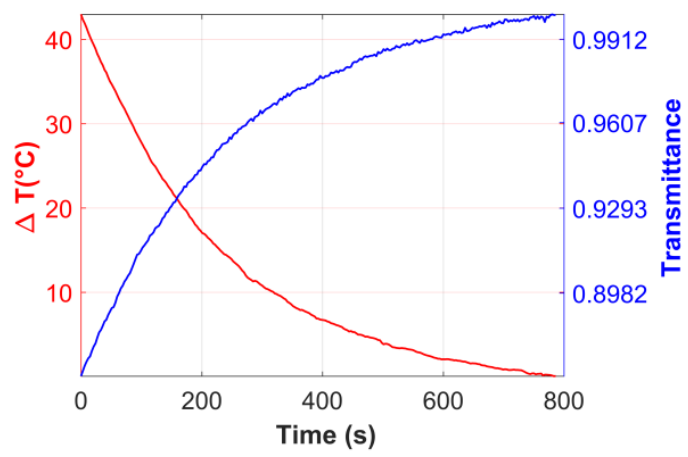

(a)

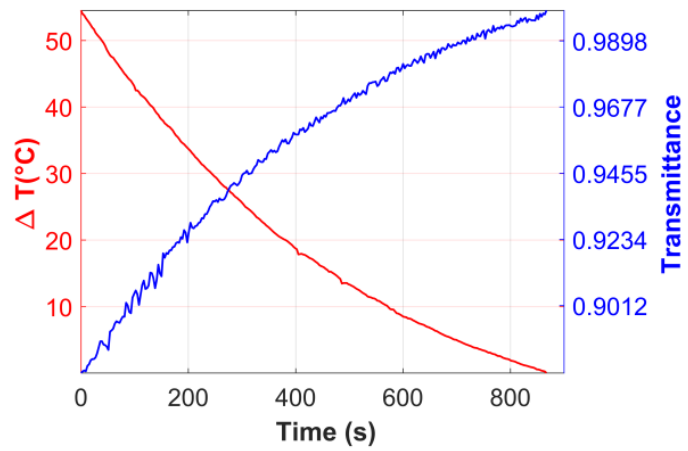

(c)

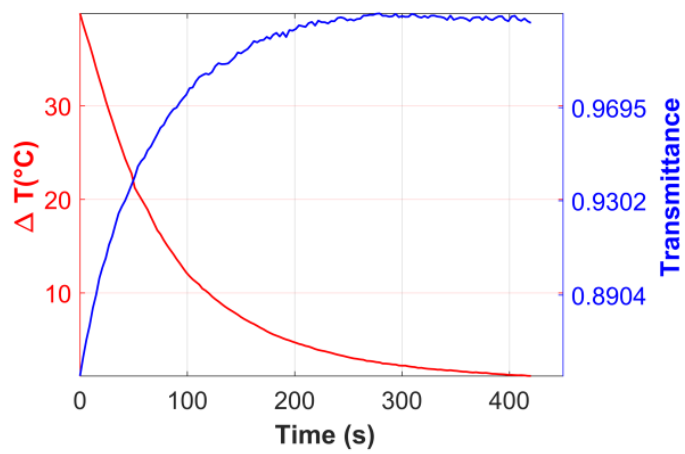

(e)

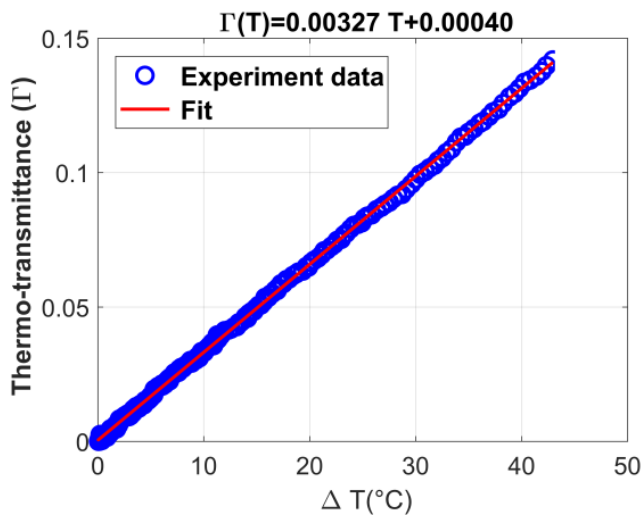

(b)

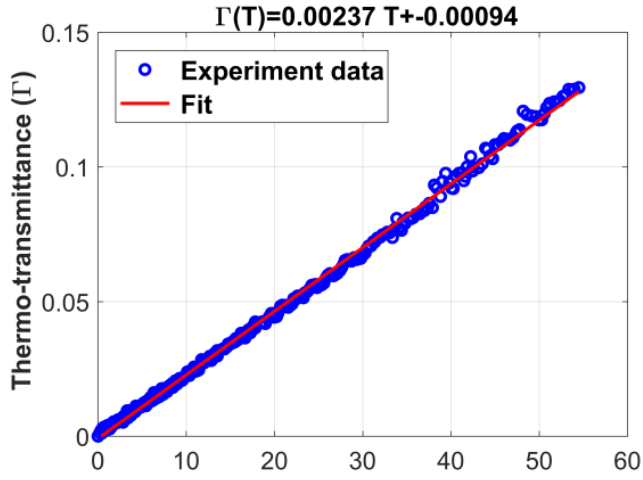

(d)

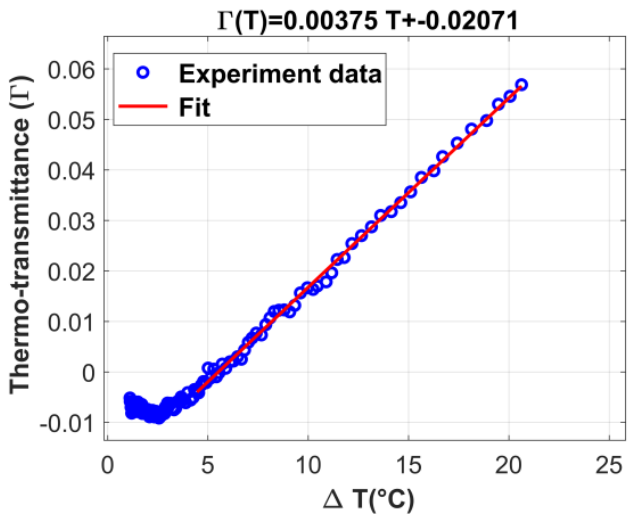

(f)

Fig. 4. Curves of transmittance $\left(I(T) / I\left(T_{0}\right)\right)$ and temperature measurement as function of time for (a) PVC, (c) PTFE (e) Wood. Calibration curve of (b) PVC (d) PTFE and (f) Wood. Circles in (b), (d) and (f): Experimental values. Continuous lines: Fitting model using equation (3). Header: Fitting equations for each sample.

The figures (4b, $4 d$ and $4 f)$ show that the noise affecting the transmittance transient case is small due the processing methods (four-image algorithm and SVD methods). However, as mentioned above, the wood attained quickly the steady state level where the calibration is not anymore valid. The calibration curves show that the thermotransmittance is linear function with transient temperature decrease. Therefore, the thermo-transmittance coefficient $\mathrm{k}$ 
can be obtained according to the Eq. (3) by dividing the slope of linear fit by the corresponding thickness for each material. The obtained values are represented in table 1:

Table 1. Thermo-transmittance with determination coefficients values of PVC, PTFE and wood

\begin{tabular}{|c|c|c|c|c|c|c|}
\hline & Slope $\left(K^{1}\right)$ & $\begin{array}{c}\text { Thermo- } \\
(m)\end{array}$ & $\begin{array}{c}\text { Thickness } \\
\text { transmittan } \\
\text { ce } \\
\text { coefficient } \\
\kappa\left(K^{1} m^{-1}\right)\end{array}$ & $\mathrm{R}^{2}$ & $\begin{array}{c}\mathrm{t}_{\mathrm{c}}=\rho \mathrm{C}_{\mathrm{p}} \mathrm{e} / \mathrm{h} \\
(\mathrm{s})\end{array}$ & $\mathrm{Bi}$ \\
\hline PTFE & $2.3710^{-3}$ & $610^{-3}$ & $\mathbf{0 . 3 9 5}$ & 0.9988 & $\mathbf{4 3 0}$ & 0.333 \\
\hline PVC & $3.2710^{-3}$ & $410^{-3}$ & $\mathbf{0 . 8 1 7}$ & 0.9994 & $\mathbf{2 9 0}$ & 0.5 \\
\hline Wood & $3.7510^{-3}$ & $310^{-3}$ & $\mathbf{1 . 2 5}$ & 0.9914 & $\mathbf{1 4 4}$ & 0.462 \\
\hline
\end{tabular}

The thermo-transmittance coefficient values found in this study are comparable with a value of similar insulating semi-transparent material (POM polymer) that is found to be around $2 \mathrm{~K}^{-1} \mathrm{~m}^{-1}[5]$. The gap between these values may due to: firstly, the nature of material and its behavior in terms of transmittance during temperature release. Secondly, in this study, the temperature is supposed constant along the thickness where the temperature has a parabolic profile. Moreover, the temperature field of the ROI is supposed homogeneous and equal to the temperature of one pixel measured by thermocouple (lumped body hypothesis). Nevertheless, it is checked numerically that the temperature difference between the center and end of ROI is low and around 2 degrees which allows validating this hypothesis. Finally, the studied materials and material used in [5] have different thermo-physical properties (density, heat capacity) and thickness. This can be noticed in table 1 where the characteristic time of materials is inversely proportional to the estimated thermo-transmittance coefficient. The factor $\left(t_{c}\right)$ represents the denominator of the exponential term in heat equation solution of lumped body. The decreasing of this factor leads to drastic decrease of temperature (short transient case) which can be shown in figure (4-e and 4-f) and thereby increase the thermo-transmittance coefficient as mentioned in bold in table 1.

Theoretically, the thermo-transmittance of homogeneous material should be the same all over the ROI but the inhomogeneity of THz beam, optical characteristic, state of surface of materials, inhomogeneity of structure (wood) and noise, lead to variable estimated coefficients. However, the histogram of distribution of this coefficient should be centered on one value. Once the thermo-transmittance coefficient is estimated using Eq. (3), the reconstruction of temperature field is then possible. The obtained results for PVC and wood at different temperature are shown in the figure 5 and 6 :

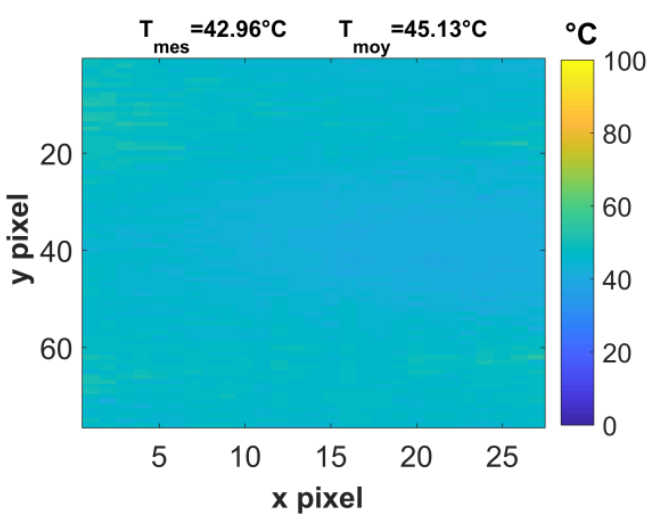

(a)

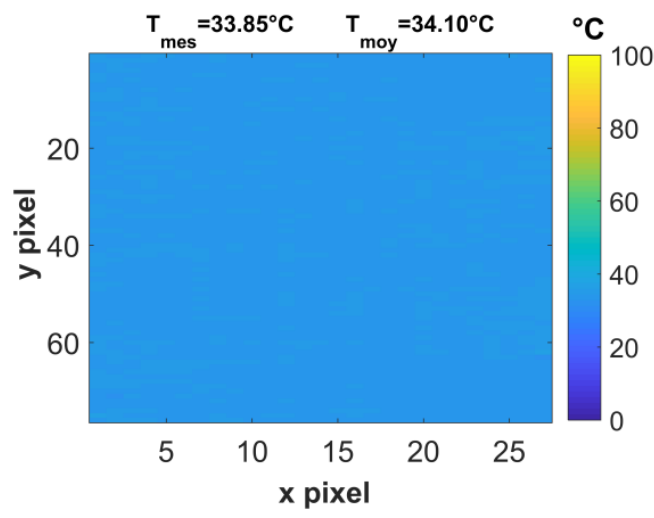

(b) 


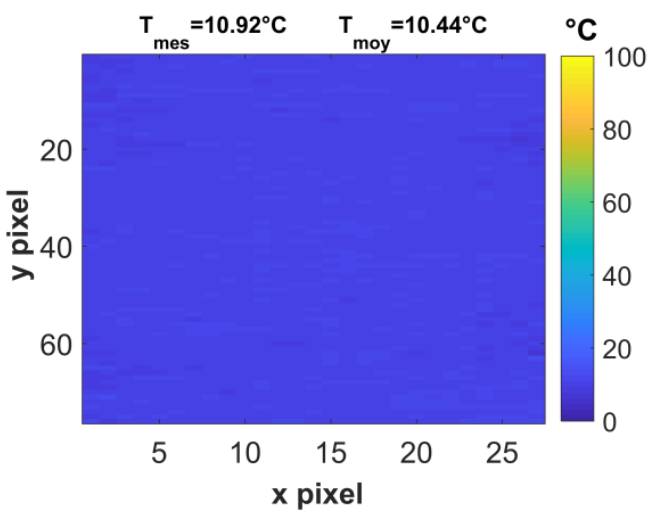

(c)

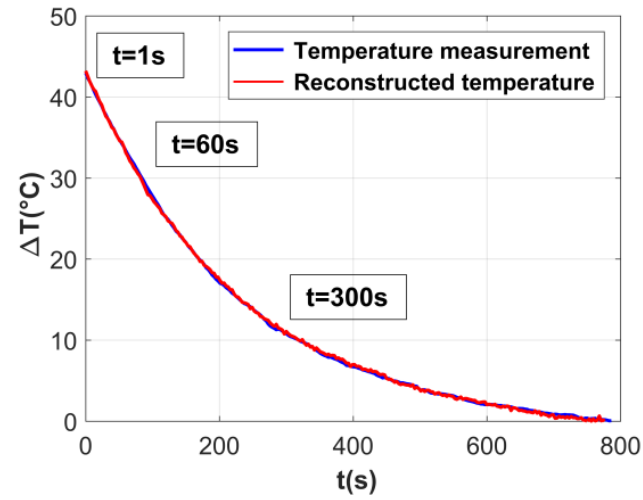

(d)

Fig. 5: Contactless temperature field measurement by THz of PVC at (a) $t=1 \mathrm{~s}$, (b) $t=60 \mathrm{~s}$, (c) $t=300 \mathrm{~s}$, (d) Transient temperature release function of time. Headers: $T_{\text {mes }}$, temperature measured by thermocouple, $T_{\text {moy, }}$ mean temperature of image. Blue line: Reconstructed temperature, red line: Temperature measurement by thermocouple.

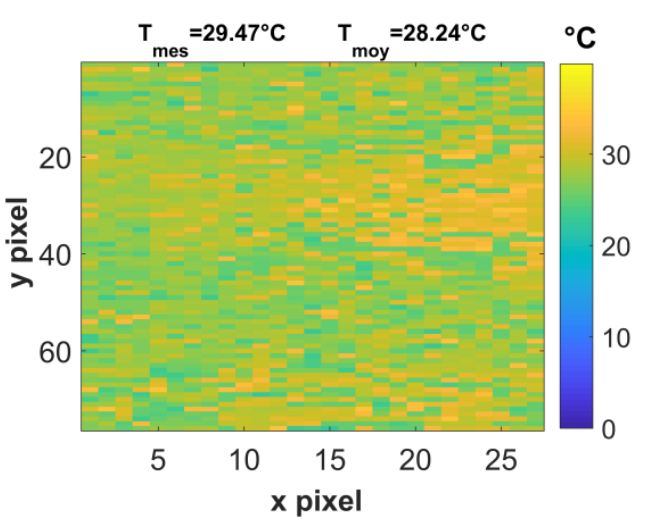

(a)

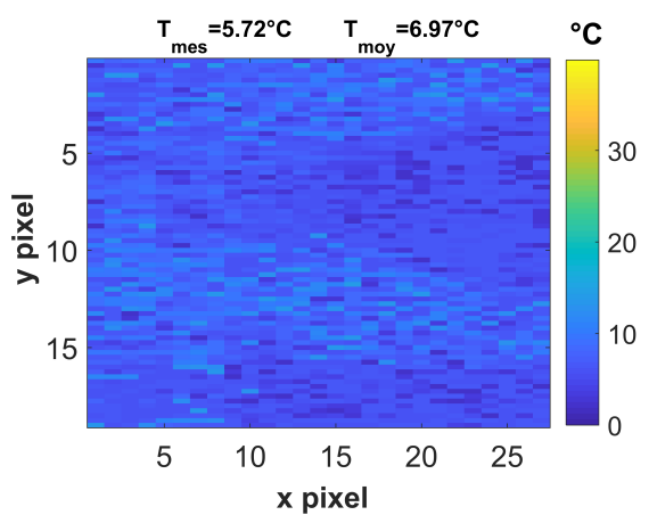

(c)

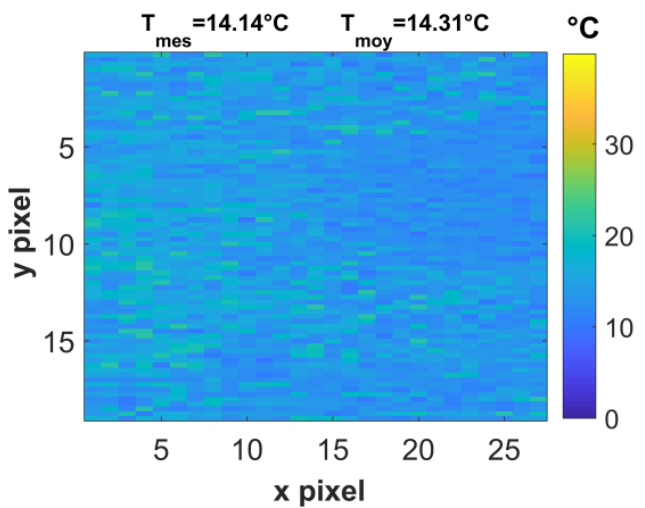

(b)

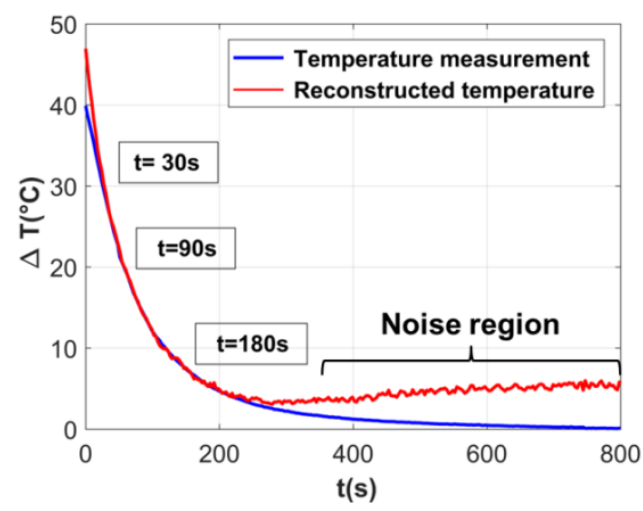

(d)

Fig. 6: Contactless temperature field measurement by $\mathrm{THz}$ of wood at (a) $t=30 \mathrm{~s}$, (b) $t=90 \mathrm{~s}$, (c) $t=180 \mathrm{~s}$, (d) Transient temperature release function of time. Headers: $T_{\text {mes }}$, temperature measured by thermocouple, $T_{\text {moy, }}$ mean temperature of image. Blue line: Reconstructed temperature, red line: Temperature measurement by thermocouple.

The figure 5 shows good estimation of temperature field for homogeneous sample such as PVC with relative error of temperature estimation of $3 \%$ for homogeneous materials. However, a heterogeneous sample such as wood scatters the temperature field but the mean value is still kept close to the punctual temperature measured by thermocouple (figure 5). 


\subsection{Determination of diffusion coefficient}

The hydric diffusion through a homogeneous paper such as blotting paper is studied using the same system and processing image mentioned above. However, the obtained images of ROI (140 x $120 \times 200)$ were averaged along the axis $y$ then resized (sub-ROI) to obtain space-time image (40 x 190) as shown in figure 7 . Thereafter, the water content can be obtained using the Eq. (6) which is indicated in the figure 6-a. On the other hand, numerical simulations of diffusion using finite difference (central scheme) with experimental boundary and initial condition were performed (figure 6-a).

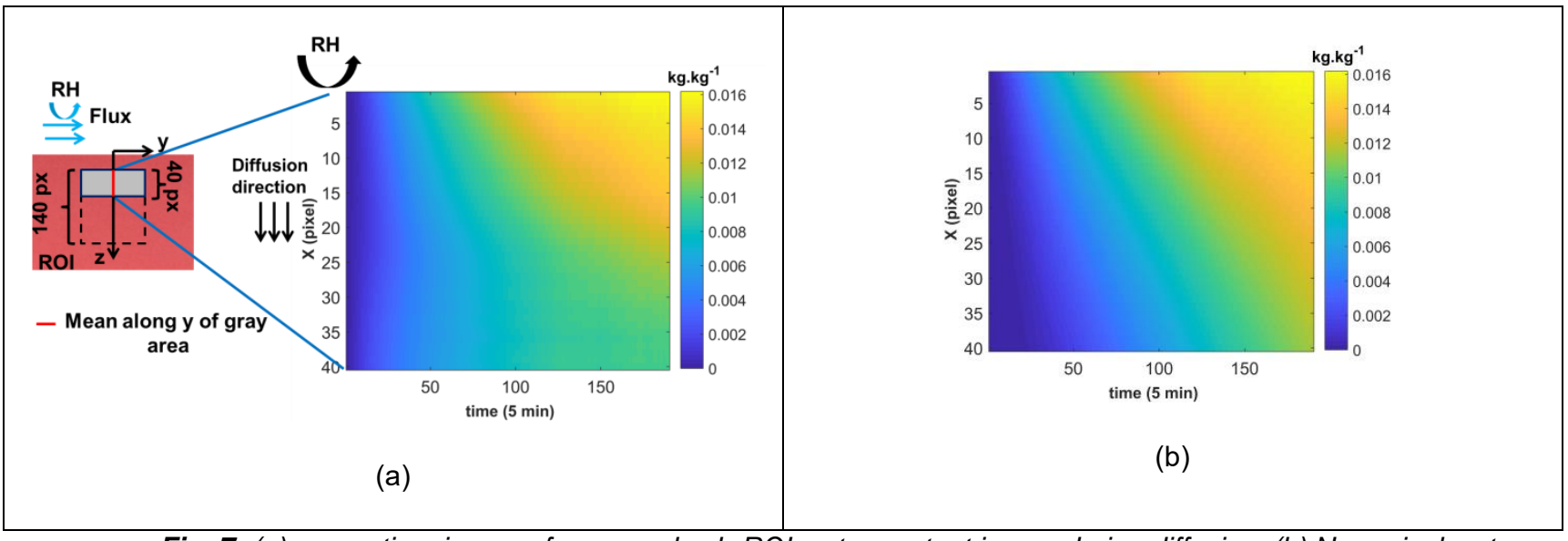

Fig. 7: (a) space-time image of averaged sub-ROI water content image during diffusion, (b) Numerical water content using boundary condition mentioned in (a) with 1 pixel $=0.25 \mathrm{~mm}$.

The water content is initially lows then increase with time due to the diffusion of vapor with time. Moreover, water content increases faster as the positon is closer to the vapor flow at the boundary which indicates the diffusion long axis $z$. Thereafter, the diffusion can be estimated by minimization between the experimental and numerical data. The figure 7 shows that the best fit should be carried out in the upper triangular part of image (yellow colored part) corresponding to the dynamic of diffusion. The blue part represents the area where no diffusion occurs yet. The Levenberg-Marquardt minimization of experimental and numerical data allows the obtaining of hydric coefficient $D$ that is of $4.210^{-8} \mathrm{~m}^{2} \cdot \mathrm{s}^{-1}$. This value is in agreement with found value in literature of blotting paper that is $\mathrm{D}=4.1510^{-8} \mathrm{~m}^{2} \cdot \mathrm{s}^{-1}[20]$. The last value has been found using $\mathrm{THz}$ technique with mono-detector but with different boundary conditions. The error between two values is around $3 \%$ which proves also the capability of this lock-in amplification-based setup for measurements of hydric diffusion.

\section{Conclusion \& perspectives}

This study showed the capability of $\mathrm{THz}$ imaging technique as a contactless tool for transient thermal and hydric measurements. First, an infrared coupled with TTC is used as full-field system to measure the transient temperature of semi-transparent materials. Measurements were performed by a lock-in technique mode with an appropriate numerical method to obtain images of transmitted amplitude (four-image algorithm). The SVD method was applied to the images of amplitude to clear noise and to enhance image quality.

Linear behavior based on Beer-Lambert law between the thermo-transmittance and the temperature gradient is checked. Indeed, the thermo-transmittance coefficient at terahertz range is 100 times more sensitive than in visible. Moreover, this coefficient is estimated for PVC, PTFE and wood that is independent of temperature can be used to estimate the transient temperature release of insulating materials. On the other hand, this system is used in transient hydric diffusion to monitor the water content with homogeneous paper in order to estimate the diffusion coefficient. The excitation is carried out through a fluidic exciter ensuring continue excitation (Echelon form). The diffusion coefficient of blotting paper is determined with an error of $3 \%$.

Finally, the use of infrared camera coupled with TTC and THz source based on lock-in amplitude amplification appears to be a promising imaging technique for the contactless measurement of the transient temperatures. Image processing methods like four-image algorithm and SVD method showed a high influence on the quality of measurement. Such results paves the way to more sophisticated $\mathrm{THz}$ applications like $\mathrm{THz}$ tomography [21] aiming to create 3D reconstructions of transient temperature fields, which is the goal of ongoing studies. For hydric application, a future study will be devoted to study the diffusion in heterogeneous materials such as wood. 


\section{REFERENCES}

[1] C. Pradere, M. Ryu, A. Sommier, M. Romano, A. Kusiak, J. L. Battaglia, J. C. Batsale, and J. Morikawa. "Noncontact temperature field measurement of solids by infrared multispectral thermotransmittance". Journal of Applied Physics 121, 085102 (2017); doi: 10.1063/1.4976209.

[2] Romano. M, Ndiaye. C, Duphil. A, Sommier A, Morikawa J, Mascetti. J, Batsale. J. C, Servant. L, Pradere. C. Fast infrared imaging spectroscopy technique (FIIST). (2015) Infrared Physics and Technology, 68, pp. 152-158.

[3] C. Pradere, J. P. Caumes, S. BenKhemis, G. Pernot, E. Palomo, S. Dilhaire and J. C. Batsale1. Thermoreflectance temperature measurement with millimeter wave. Review of scientific instruments 85,064904 (2014)

[4] M. Romano, A. Sommier, J. C. Batsale and C. Pradere. 3D transient temperature measurement in homogeneous solid material with THz waves. SPIE Photonics Europe, 2016, Brussels, Belgium.

[5] Cyndie Poulin, Meriam Triki, Karim Bousmaki, Alexandre Duhant, Hervé Louche and Bertrand Wattrisse. "Terahertz thermometry system to measure temperature in the thickness of a solid polymer". Quantitative InfraRed Thermography Journal. TQRT 2016-0032.

[6] Sergey V. Dvinskikh, Marielle Henriksson, Antonio Lorenzo Mendicino, Stefania Fortino, Tomi Toratti. " NMR imaging study and multi-Fickian numerical simulation of moisture transfer in Norway spruce samples". Engineering Structures 33 (2011) 3079-3086.

[7] Marjan Sedighi Gilani, Saeed Abbasion, Eberhard Lehmann, Jan Carmeliet, Dominique Derome. "Neutron imaging of moisture displacement due to steep temperature gradients in hardwood". International Journal of Thermal Sciences 81 (2014) 1-12.

[8] Stephane Hameury \& Magdalena Sterley. "Magnetic resonance imaging of moisture distribution in pinus sylvestris I exposed to daily indoor relative humidity fluctuations ". Wood Material Science and Engineering, 2006; 1: 116-126.

[9] Boris Clouet, Régis Pommier and Michel Danis. "New composite timbers, full field analysis of adhesive behavior". Part of the series Conference Proceedings of the Society for Experimental Mechanics Series pp 51-58. 14 August 2013.

[10] M. Bensalem, A. Sommier, J. C. Mindeguia, J. C. Batsale and C. Pradere. Terahertz Measurement of the Water Content Distribution in Wood Materials. J Infrared Milli Terahz Waves, Nov 2017, DOI 10.1007/s10762-017-0441-7.

[11] Jeffrey White and David Zimdars. "Time Domain Terahertz Non Destructive Evaluation of Water Intrusion in Composites and Corrosion under Insulation". (C) 2007 Optical Society of America.

[12] Christian Jansen, Steffen Wietzke, Ole Peters, Maik Scheller, Nico Vieweg, Mohammed Salhi, Norman Krumbholz, Christian Jördens, Thomas Hochrein and Martin Koch. "Terahertz imaging: application and perspectives". (c) 2007 Optical Society of America. Applied Optics/ vol. 49, NO, 19/1 July 2010.

[13] Dr GUNTER Urbasch, "Monitoring the plant water status with terahertz waves". $4^{\text {th }}$ Terahertz workshop on Elba (2012).

[14] John F. Federici. "Review of Moisture and Liquid Detection and Mapping using Terahertz Imaging". J Infrared Milli Terahz Waves (2012) 33:97-126.

[15] D. Banerjee, W. von Spiege, M. D. Thomson, S. Schabel, H.G.Roskos. "Diagnosing water content in paper by terahertz radiation". 9 June 2008 / Vol. 16, No. 12 / OPTICS EXPRESS 9060.

[16] Takeshi Yasui, Takashi Yasuda, Ken-ichi Sawanaka, and Tsutomu Araki. "Terahertz paintmeter for noncontact monitoring of thickness and drying progress in paint film". (c) 2005 Optical Society of America. 10 November 2005.

[17] M. Romano, A. Chulkov, A. Sommier, D. Balageas, V. Vavilov, J. C. Batsale, C. Pradere. "Broadband Subterahertz Camera Based on Photothermal Conversion and IRThermography". Journal of Infrared Millimeter and Terahertz waves. January 2016. Doi: 10.1007/s10762-015-0241-x.

[18] J. M. Rampnoux, H. Michel, M. A. Salhi, S. Grauby, W. Claeys, S. Dilhaire. "Time gating imaging through thick silicon substrate : a new step towards backside characterization". Microelectronics Reliability 46 (2006) 15201524. doi:10.1016/j.microrel. 2006.07.029.

[19] MATLAB FVT®*: Copyright (c) 2012, 2013, 2014, 2015 Ali Akbar Eftekhari All rights reserved.

[20] D. DESROSIERS, (2009) Modélisation du transfert de chaleur et d'humidité dans une membrane de cellulose, Mémoire de maîtrise, Université du Québec (Trois-Rivières, MONTRÉAL).

[21] Saroj R. Tripathi, yuya Sugiyama, Kosuke Murate, Kazuki Imayama and Kodo Kawase. "Teraherz wave threedimensional computed tomography based on injection-seeded terahertz wave parametric emitter". Optics Express March 2016. DOI:10.1364/ OE.24.006433. 